\title{
Bioanalysis
}

\section{White Paper on Recent Issues in Bioanalysis: Chromatographic Assays (Part 1 - Innovation in Small Molecules and Oligonucleotides \& Mass Spectrometric Method Development Strategies for Large Molecule Bioanalysis)}

Christine Fandozzi ${ }^{\ddagger, 1}$, Christopher Evans ${ }^{\ddagger, 2}$, Amanda Wilson ${ }^{\ddagger}{ }^{\ddagger}$, Dian Su ${ }^{\ddagger}, 4$, Melanie Anderson ${ }^{\ddagger}, 5$, Valerie Clausen ${ }^{\ddagger}, 6$, Lieve Dillen ${ }^{\ddagger}$, , Fabio Garofolo*, ${ }^{\star}, 8$, Chris Holliman ${ }^{\ddagger}$, Elliott Nickbarg ${ }^{\ddagger 10}$, Timothy Olah ${ }^{\ddagger 11}$, Ragu Ramanathan ${ }^{\ddagger}, 9$, Hui Zhang ${ }^{\ddagger}, 9$, Surinder Kaur $^{\S, 4}$, Renuka Pillutla ${ }^{\S, 11}$, Hongbin $\mathrm{Yu}^{\S, 12}$, Kevin Bateman ${ }^{\S, 1}$, Lorella Di Donato ${ }^{\S, 13}$, Shawna Hengel ${ }^{\S, 14}$, Wenying Jian ${ }^{\S, 15}$, Barry Jones ${ }^{\S, 16}$, John Kellie ${ }^{\S, 2}$, Anita Lee ${ }^{\S, 17}$, Joe Palandra ${ }^{\S, 18}$, Natasha Savoie ${ }^{\S, 19}$, Petia Shipkova ${ }^{\S, 11}$, Susan Spitz ${ }^{\S, 20}$, Dian Su ${ }^{\S, 4}$, Matthew Szapacs ${ }^{\S, 2}$, Jian Wang ${ }^{\S, 11}$, Katherine Wright ${ }^{\S, 18}$ \& Jianing Zeng ${ }^{\S, 11}$

\footnotetext{
${ }^{1}$ Merck, West Point, PA, USA

${ }^{2}$ GlaxoSmithKline, Collegeville, PA, USA

${ }^{3}$ Clinical Pharmacology \& Safety Sciences, Translational

Biomarker \& Bioanalysis, BioPharmaceuticals R\&D, AstraZeneca, Cambridge, UK

${ }^{4}$ Genentech, South San Francisco, CA, USA

${ }^{5}$ Merck, Philadelphia, PA, USA

${ }^{6}$ Alnylam Pharmaceuticals, Cambridge, MA, USA

${ }^{7}$ Janssen Research \& Development, Beerse, Belgium

${ }^{8}$ Angelini Pharma, Pomezia, RM, Italy

${ }^{9}$ Pfizer, Groton, CT, USA
}

\author{
${ }^{10}$ Merck, Boston, MA, USA \\ ${ }^{11}$ Bristol-Myers Squibb, Princeton, NJ, USA \\ ${ }^{12}$ Boehringer-Ingelheim, Ridgefield, CT, USA \\ ${ }^{13}$ Caprion, Montreal, QC, Canada \\ ${ }^{14}$ Seattle Genetics, Inc., Bothell, WA, USA \\ ${ }^{15}$ Janssen Research \& Development, Spring House, PA, USA \\ ${ }^{16}$ Q2 Solutions, Ithaca, NY, USA \\ ${ }^{17}$ Merck, Kenilworth, NJ, USA \\ ${ }^{18}$ Pfizer, Andover, MA, USA \\ ${ }^{19}$ CFABS, Montreal, QC, CAN \\ ${ }^{20}$ Incyte, Wilmington, DE, USA
}

The $201913^{\text {th }}$ Workshop on Recent Issues in Bioanalysis (WRIB) took place in New Orleans, LA, USA on April 1-5, 2019 with an attendance of over 1000 representatives from pharmaceutical/biopharmaceutical companies, biotechnology companies, contract research organizations and regulatory agencies worldwide. WRIB was once again a 5-day, week-long event - a full immersion week of bioanalysis, biomarkers, immunogenicity and gene therapy. As usual, it was specifically designed to facilitate sharing, reviewing, discussing and agreeing on approaches to address the most current issues of interest including both small- and large-molecule bioanalysis involving LCMS, hybrid LBA/LCMS, LBA cell-based/flow cytometry assays and qPCR approaches. This 2019 White Paper encompasses recommendations emerging from the extensive discussions held during the workshop, and is aimed to provide the bioanalytical community with key information and practical solutions on topics and issues addressed, in an effort to enable advances in scientific excellence, improved quality and better regulatory compliance. Due to its length, the 2019 edition of this comprehensive White Paper has been divided into three parts for editorial reasons. This publication (Part 1) covers the recommendations on Innovation in Small Molecules and Oligonucleotides \& Mass Spec Method Development Strategies for Large Molecules Bioanalysis. Part 2 (2018 FDA BMV Guidance, 2019 ICH M10 BMV Draft Guideline and regulatory agencies' input on bioanalysis, biomarkers, immunogenicity and gene therapy) and Part 3 (New Insights in Biomarkers Assays Validation, Current \& Effective Strategies for Critical Reagent Management, Flow Cytometry Validation in drug discovery \& development \& CLSI H62, Interpretation of the 2019

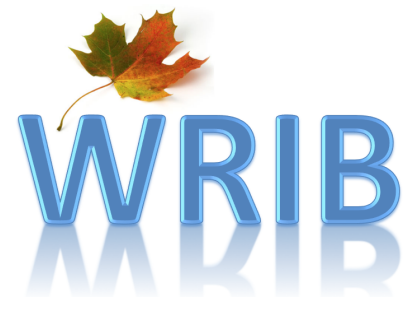

¥SECTION 1 - Innovation in Small Molecules and Oligonucleotides (authors in Section 1 are presented in alphabetical order of their last name, with the exception of the first four authors who were session chairs, working dinner facilitators, major contributors and/or note-takers). §SECTION 2 - Mass Spectrometric Method Development Strategies for Large Molecule Bioanalysis (authors in Section 2 are presented in alphabetical order of their last name, with the exception of the first three authors who were session chairs and/or working dinner facilitators).

Disclaimer: The views expressed in this article are those of the authors and do not reflect official policy of the US FDA, Europe EMA, France ANSM, UK MHRA, Brazil ANVISA, Health Canada, Japan MHLW, and Norway NoMA. No official endorsement by the FDA, EMA, ANSM, Health Canada, MHRA, ANVISA, MHLW or NoMA is intended or should be inferred.

*Author for correspondence: fabiogarofolo@hotmail.com 
FDA Immunogenicity Guidance and The Gene Therapy Bioanalytical Challenges) are published in volume 11 of Bioanalysis, issues 23 and 24 (2019), respectively.

First draft submitted: 13 September 2019; Accepted for publication: 19 October 2019; Published online: 6 December 2019

\section{Index}

- Introduction

- SECTION 1 - Innovation in Small Molecules \& Oligonucleotides

- Discussion Topics

- Discussions, Consensus \& Conclusions

- Recommendations

- SECTION 2 - Mass Spectrometric Method Development Strategies for Large Molecule Bioanalysis

- Discussion Topics

- Discussions, Consensus \& Conclusions

- Recommendations

- References

\section{Acronyms}

\begin{tabular}{|c|c|}
\hline ADC: & Antibody-drug conjugate \\
\hline ADE: & Acoustic droplet ejection \\
\hline ADME: & Absorption, distribution, metabolism, excretion \\
\hline AEX: & Anion exchange \\
\hline AMS: & Accelerator mass spectrometry \\
\hline AQUA: & Absolute quantification \\
\hline ASMS: & Affinity selection mass spectrometry \\
\hline ASO: & Antisense oligonucleotides \\
\hline BAV: & Biomarker assay validation \\
\hline BMV: & Bioanalytical method validation \\
\hline CLSI: & Clinical \& Laboratory Standards Institute \\
\hline CoA: & Certificate of analysis \\
\hline CRO: & Contract research organization \\
\hline CSF: & Cerebrospinal fluid \\
\hline DAR: & Drug antibody ratio \\
\hline DDI: & Drug-drug interaction \\
\hline DMPK: & Drug metabolism and pharmacokinetics \\
\hline DNA: & Deoxyribonucleic acid \\
\hline DMS: & Differential mobility spectrometry \\
\hline ELISA: & Enzyme-linked immunosorbent assay \\
\hline ESI: & Electrospray ionization \\
\hline HFIP: & Hexafluoroisopropanol \\
\hline HPLC: & High performance liquid chromatography \\
\hline HRMS: & High resolution mass spectrometry \\
\hline IA: & Immunoaffinity \\
\hline ICD: & Informed consent document \\
\hline IND: & Investigational new drug \\
\hline IS: & Internal standard \\
\hline LBA: & Ligand binding assay \\
\hline LC: & Liquid chromatography \\
\hline
\end{tabular}




\begin{tabular}{|c|c|}
\hline LCM: & Life cycle management \\
\hline LCMS: & Liquid chromatography mass spectrometry \\
\hline mAb: & Monoclonal antibody \\
\hline MATE: & Multidrug and toxin extrusion proteins \\
\hline miRNA: & Micro ribonucleic acid \\
\hline MIST: & Metabolites in safety testing \\
\hline MRM: & Multiple reaction monitoring \\
\hline MS: & Mass spectrometry \\
\hline ncRNA: & Non-coding ribonucleic acid \\
\hline OPP: & Open-port probe \\
\hline PD: & Pharmacodynamics \\
\hline PDC: & Protein-drug conjugate \\
\hline PK: & Pharmacokinetics \\
\hline PROTAC: & Proteolysis targeting chimera \\
\hline QC: & Quality control \\
\hline RNA: & Ribonucleic acid \\
\hline RP-IP-LCMS: & Reverse phase-ion pairing liquid chromatography mass spectrometry \\
\hline SiRNA: & Small interfering ribonucleic acid \\
\hline SIL: & Stable isotope label \\
\hline SPE: & Solid phase extraction \\
\hline SRM: & Selected reaction monitoring \\
\hline TM: & Target modulation \\
\hline TQMS: & Triple quadrupole mass spectrometry \\
\hline UHPLC: & Ultra-high performance liquid chromatography \\
\hline WRIB: & Workshop on Recent Issues in Bioanalysis \\
\hline
\end{tabular}

\section{Introduction}

The $13^{\text {th }}$ edition of the Workshop on Recent Issues in Bioanalysis (13 $3^{\text {th }}$ WRIB) was held in New Orleans, LA, USA, on April 1-5, 2019 with an attendance of over 1000 representatives from pharmaceutical/biopharmaceutical companies, biotechnology companies, contract research organizations, and regulatory agencies worldwide. The workshop included three sequential main workshop days, six additional full-day training sessions that together spanned an entire week in order to allow exhaustive and thorough coverage of all major issues in bioanalysis, biomarkers, immunogenicity and gene therapy.

As in previous years, this year's WRIB continued to gather a wide diversity of international industry opinion leaders and regulatory authority experts working on both small and large molecules to facilitate sharing and discussions focused on improving quality, increasing regulatory compliance and achieving scientific excellence on bioanalytical issues.

The active contributing chairs included Dr Christine Fandozzi (Merck), Dr Christopher Evans (GlaxoSmithKline), Dr Brian Booth (US FDA), Dr Renuka Pillutla (Bristol-Myers Squibb), Dr Fabio Garofolo (Angelini Pharma), Dr Becky Schweighardt (BioMarin), Dr Meina Liang (AstraZeneca), and Dr Lauren Stevenson (Biogen).

The participation of regulatory agency representatives continued to grow at WRIB [1-21] including the below:

- Regulated Bioanalysis: Dr Sean Kassim (US FDA), Dr Sam Haidar (US FDA), Dr Seongeun (Julia) Cho (US FDA), Dr John Kadavil (US FDA), Dr Arindam Dasgupta (US FDA), Dr Brian Booth (US FDA), Dr Sriram Subramaniam (US FDA), Dr Theingi Thway (US FDA), Dr Nilufar Tampal (US FDA), Dr Jan Welink (EU EMA), Dr Olivier Le Blaye (France ANSM), Mr Stephen Vinter (UK MHRA), Ms Emma Whale (UK MHRA), Dr Anna Edmison (Health Canada), Dr Catherine Soo (Health Canada), Mr Gustavo Mendes Lima Santos (Brazil ANVISA), Ms Thais Correa Rocha (Brazil ANVISA); 
- Biomarkers: Dr Yow-Ming Wang (US FDA), Dr Abbas Bandukwala (US FDA), Dr Kevin Maher (US FDA), Dr Shashi Amur (US FDA), Dr Shirley Hopper (UK MHRA), Dr Yoshiro Saito (Japan MHLW-NIHS);

- Immunogenicity: Dr João Pedras-Vasconcelos (US FDA), Dr Haoheng Yan (US FDA), Dr Susan Kirshner (US FDA; remote), Dr Daniela Verthelyi (US FDA; remote), Dr Elana Cherry (Health Canada), Dr Akiko Ishii-Watabe (Japan MHLW-NIHS), Dr Venke Skibeli (Norway NoMA), Dr Therese Solstad Saunders (Norway NoMA);

- Gene Therapy: Dr Nirjal Bhattarai (US FDA), Dr Heba Degheidy (US FDA).

The $13^{\text {th }}$ WRIB was designed to cover a wide range of topics in bioanalysis, biomarkers, immunogenicity and a special full-day session dedicated to gene therapy bioanalytical challenges. Moreover, the $13^{\text {th }}$ WRIB included daily working dinners and lectures from both industry experts and regulatory representatives, which culminated in open panel discussions amongst the presenters, regulators and attendees in order to reach consensus on items presented in this White Paper.

While the $13^{\text {th }}$ WRIB continued its traditional emphasis on method development challenges and novel solutions in bioanalysis, it also included an in-depth focus on the recently released ICH M10 BMV Draft Guideline [22]. Three full sessions, two working dinners and three open forums were dedicated to cover the hot topics of the ICH M10 BMV Draft Guideline, and to actively interact with the regulators' expert panel and work together as a Global Bioanalytical Community with the goal to provide official comments on the ICH M10 BMV draft guideline. Harmonized topics among US FDA, EU EMA, Health Canada, Japan MHLW and Brazil ANVISA regulations, unresolved issues and on-going industry/regulator discussions were thoroughly evaluated to support the regulatory requirements of the ICH M10 BMV Draft Guideline which, when finalized, will supersede the regional guidance of the participating health authorities.

The three sessions and open forums on ICH M10 activities at WRIB were organized and coordinated by Dr Brian Booth (US FDA, ICH M10 EWG Regulatory Chair), Dr Jan Welink (EU EMA), Dr Anna Edmison (Health Canada), Dr Akiko Ishii-Watabe (MHLW, ICH M10 EWG Rapporteur), Dr Yoshiro Saito (MHLW), and Ms Thais Correa Rocha (ANVISA) and with input and active participation from numerous industry/regulator opinion leaders.

A total of 48 recent issues ('hot' topics) were addressed and distilled into a series of relevant recommendations. Presented in the current White Paper is the background on each issue, exchanges, consensus and resulting recommendations on these 48 topics.

Due to its length, the 2019 edition of this comprehensive White Paper has been divided into three parts for editorial reasons. This publication covers Part 1 recommendations.

Part 1 - Bioanalysis Volume 11, Issue 22 (November 2019)

Innovation in Small Molecules \& Oligonucleotides:

- Novel Therapeutic Modalities (two topics);

- Innovation in Small Molecules (three topics);

- Small Molecule Biomarkers by LCMS (one topic);

- Oligonucleotides (one topic).

Mass Spectrometric Method Development Strategies for Large Molecules Bioanalysis:

- Innovation in Hybrid LBA/LCMS Assays (five topics);

- Biomarker Assays (three topics).

Part 2 - Bioanalysis Volume 11, Issue 23 (December 2019)

Implementation of 2018 FDA BMV Guidance (ten topics):

Industry/Regulators' Feedback on ICH M10 BMV Draft Guidelines (14 topics).

Input from Regulatory Agencies on Bioanalysis \& BMV.

Input from Regulatory Agencies on Immunogenicity \& Biomarkers.

Part 3 - Bioanalysis Volume 11, Issue 24 (December 2019)

New Insights in Biomarker Assay Validation (BAV): 
- Fit for Purpose \& Context of Use (two topics);

- Free Assays (one topic);

- BAV Guidelines (two topics).

Current \& Effective Strategies for Critical Reagent Management:

- Characterization \& Stability (three topics);

- Life Cycle Management (one topic);

- Flow Cytometry (one topic);

- Challenges \& Approaches (one topic).

Flow Cytometry Validation in Drug Discovery \& Development \& CLSI H62:

- Regulatory Expectations \& Validation (two topics);

- Challenges \& Approaches (three topics);

- Data Analysis (two topics).

Interpretation of the 2019 FDA Immunogenicity Guidance:

- Drug Tolerance (one topic);

- Critical Reagent \& Positive Control Characterization (one topic);

- LCM \& Clinical Relevance of ADA (one topic);

- Challenges \& Approaches (three topics);

- Cut Points \& the FDA Immunogenicity Guidance (one topic).

Gene Therapy Bioanalytical Challenges:

- Approaches to Gene Therapy Bioanalysis (four topics);

- Vaccines (one topic);

- Challenges (two topics);

- Immunogenicity (one topic).

\section{SECTION 1 - Innovation in Small Molecules \& Oligonucleotides}

Christine Fandozzi ${ }^{1}$, Christopher Evans ${ }^{2}$, Amanda Wilson ${ }^{3}$, Dian Su ${ }^{4}$, Melanie Anderson $^{5}$, Valerie Clausen ${ }^{6}$, Lieve Dillen ${ }^{7}$, Fabio Garofolo ${ }^{*}$, , Chris Holliman ${ }^{9}$, Elliott Nickbarg ${ }^{10}$, Timothy Olah $^{11}$, Ragu Ramanathan ${ }^{9}$, Hui Zhang ${ }^{9}$

Authors in Section 1 are presented in alphabetical order of their last name, with the exception of the first four authors who were session chairs, working dinner facilitators, major contributors and/or note-takers. Author affiliations can be found at the beginning of the article.

\section{Discussion topics \& consolidated questions collected from the Global Bioanalytical Community}

The following paragraphs report the consolidated questions collected from the Global Bioanalytical Community. Seven discussion topics were extracted from these questions and considered as the most relevant 'hot topics'. They were reviewed by internationally recognized opinion leaders before being submitted for discussion during the $13^{\text {th }}$ WRIB. The background on each issue, discussions, consensus and conclusions are in the next section and a summary of the key recommendations is provided in the final section of this manuscript.

\section{Novel Therapeutic Modalities}

Return of the Small Molecules

How is the optimal PK profile of novel therapeutic modalities such as PROTAC determined? Which form (free, total, bound complex) and what biological matrix is required to measure a novel therapeutic? What is the best approach to assess and manage the optimal $\mathrm{PD}$ /toxicity profile of a novel therapeutic strategy like target protein degradation: quantification of target, bystander, and/or off-target protein levels; assessment of toxicity and/or downstream effects? What level of detail will be required to convince regulatory agencies that a novel therapeutic strategy is acceptable? What are promising areas regarding the status of RNA as a target for small molecule drug discovery (ribocil-like small molecules)? What are the most promising hit-to-lead strategies for RNA targets? What 
are the main advantages of using ASMS for the characterization of selective RNA-binding small molecules? What are alternative RNA screening approaches? What are appropriate follow-up strategies to characterize small molecule hits to RNA from screening approaches?

\section{Complex Small Molecule Formulations}

In the nanomaterial draft guidance [23], what is meant by 'indirectly derived'? Can you measure two of the three components (total, free and nanomaterial associated drug) and calculate the third component? When would this be acceptable or does this mean measure a metabolite as a surrogate? Has there been any experience with the 'mathematical' approach? Nanomedicine formulations are designed to leak or rapidly break down. When the formulation is used to prepare QCs for monitoring of release during sample preparation, have the pharmaceutical sciences measurements been different to those determined in the bioanalytical laboratory? If so, how are the differences handled between documented released amounts of drug in formulations and prepared nanomedicines and $\mathrm{t}=0$ stability or baseline measurements for QC preparation? How are companies monitoring the fate of the carrier? To establish the biological fate of the carrier, is it acceptable to measure one component of the carrier as a surrogate? Is there experience in analytical approaches to measure the intact carrier in biological matrices and if yes, how? In the pre-clinical space, can this be measured once in a DMPK biodistribution study? Is there experience measuring the protein corona for a nanomaterial? If yes, what are the analytical approaches and how is this information being used? Are there particular nanomedicines where the protein corona measurement does not make sense or is not possible, for example, when components of the nanomaterial are rapidly shed in vivo?

\section{Innovation in Small Molecules Microflow-LCMS Assays}

Are we ready to replace traditional AMS with microflow-LCMS assays for microdosing? What are the method development challenges in using microflow-LCMS for microdosing absolute bioavailability studies? Are microflowLCMS assays robust enough for routine sample analysis?

\section{Acoustic Dispensing - Mass Spectrometry (AD-MS)}

What are advantages of the ADE-OPP-MS platform, as well as major limitations (sensitivity limitations, stability limitations, i.e., isomers/labile metabolite with in source fragmentation such as glucuronidation and matrix interference, etc.)? Focusing on bioanalysis applications, how broadly can we apply ADE-OPP-MS? In what scenarios may LC be skipped? How are the challenges addressed to make ADE-OPP-MS more powerful and applicable for drug discovery and development? Should DMS/ion mobility or other orthogonal separation techniques be added for all bioanalysis applications with ADE-OPP-MS?

\section{Patient Centric Microsampling}

Does collecting bioanalytical data in late stage trials add value for drug development safety and efficacy decisions? What are the potential uses for $\mathrm{PK} / \mathrm{PD} / \mathrm{TM}$ at home sampling? Is there any agreement between different regulatory agencies on the best way to implement alternative sampling? At what point should sponsors engage with regulators on their strategies for alternative sampling in a program? What are the best approaches, both bioanalytical and logistical, for implementation of alternative sampling approaches? How is sample integrity ensured in more patient centric sampling approaches, such as at home sampling?

\section{Small Molecule Biomarkers by LCMS HRMS for Small Molecule Biomarkers}

What are the main pharma/regulatory concerns with the use of endogenous biomarkers for supporting or refuting in vitro DDI predictions? With appropriate language/permissions in the ICDs and/or clinical protocols, would there be any concerns for using leftover PK samples (e.g., with appropriate storage) for endogenous biomarker profiling or DDI projections? Should parallelism tests using authentic versus surrogate matrix or surrogate analyte always be part of the endogenous biomarker qualification/validation plans? Would there be any concerns in using multiplexed HRMS assays for profiling multiple endogenous biomarkers or have the options to retrospective interrogation of raw data for newly discovered biomarkers (provided adequate language in the ICDs)? 


\section{Oligonucleotides}

Unique Strategies in Oligonucleotides

How do you control co-elution of diastereoisomers but still separate the truncated metabolites in chromatographic based assays (AEX-HPLC and RP-IP-LCMS)? Probe design in AEX-fluorescence assays is critical but seems to be trial and error (depending on the choices made for synthesis for different probes in method development). How are the correct probes designed? Is there an alternative for ion pairing and HFIP combinations in LCMS? Oligonucleotide biodistribution methodologies that include extraction are challenging. Can these be improved? Is limited recovery acceptable if acceptance criteria of accuracy and precision are met? What is needed and what is currently missing regarding metabolite identification following the 2018 White Paper recommendation on MIST? How relevant are plasma metabolites in view of the intracellular action of oligonucleotides? Oligonucleotide shortmers are often only observed in trace amounts in plasma and/or tissue; is synthesis then required?

\section{Discussions, consensus \& conclusions}

\section{Novel Therapeutic Modalities}

Return of the Small Molecules

\section{Targeted Protein Degradation}

As our understanding of the complexities of disease progression improves, innovative patient treatment therapies employing combinations of drug substances has shown substantial success in battling cancer, heart, and autoimmune diseases. The discovery and development of various biological entities and chemotypes spanning from small molecules and millamolecules to complex biotherapeutics has offered unique structural modalities to interact with specific targets whose sites are found across a variety of biological locations where diseases are manifested. Accessibility for specific binding to unique targets with subsequent alteration of pharmacologic activity is the basis for effective pharmaceutical treatment. Although biologics have garnered much of the attention recently, there are added challenges that preclude the expanded use of these agents in the treatment of other diseases. This is primarily due to their inability to reach targets at specific locations in the body combined with difficulties to reach more patients due to their cost and dosing paradigms, alternative strategies are being developed.

Small molecule therapeutics were never abandoned as treatment for other therapeutic areas, since they are less expensive to manufacture and dose, distribute well to targets, and typically are non-immunogenic. However, small molecule drugs are undergoing a re-evaluation of sorts, creatively being incorporated in complex bispecific modalities in emerging disease treatment strategies such as targeted protein degradation or homeostasis. For example, PROTACs are emerging as a potential strategy in the treatment of certain diseases, but present bioanalytical challenges during the course of their development, both in terms of evaluating their PK/ADME properties and in assessing the PD impact. However, the initial bioanalytical strategy should be similar to that employed for small molecules and can evolve, as needed. Toxicology studies can also be conducted in a similar manner to small molecules by measuring typical TK markers and toxicology readouts (e.g., histopathology). If there are significant findings, mechanistic studies such as off-target activities can be explored on a case by case basis. The practicality of PROTAC will be confirmed by assessing the $\mathrm{PK} / \mathrm{PD} /$ toxicology data but this approach needs further exploration until proven safety and efficacy data convinces regulatory agencies that a novel therapeutic strategy is acceptable.

PROTACs are high molecular weight $(\sim 1000 \mathrm{MW})$ small molecules, are prone to non-specific binding to glass and plastic and are analyzed by means of LCMS, typically using low ionization energy to ensure no in-source fragmentation. It was concluded that when measuring a PROTAC, mechanistically free, total, and bound complexes are of interest in discovery and it was acknowledged that the PROTAC bound complexes are very dynamic. It was recommended to measure target knockdown in tumor tissue from an animal model. When performing regulated bioanalysis, measurement of total PROTAC concentration in plasma or accessible tissues (e.g., tumor biopsies) is recommended.

\section{RNA-Based Drug Target Therapeutics}

Approximately $60 \%$ of DNA is transcribed as RNA, but only $1-2 \%$ codes for proteins. Genetic linkage studies of single nucleotide polymorphisms show that the numbers of non-coding RNAs (ncRNAs) involved in cellular processes are similar to the numbers of protein coding genes. The function of ncRNA and its interactions with small molecules is relatively unexplored, and understanding the druggability of ncRNAs with small molecules will likely open up new therapeutic approaches for various diseases. It was also discussed that G-quadruplex RNAs could be the target for drug binding. Literature has shown that RNA can be the target for small molecule drug 
discovery [24]. It was agreed that the key to these modalities is the binding selectivity. Further work on defining disease-relevant druggable RNA targets is also necessary.

\section{Complex Small Molecule Formulations}

Novel drug delivery systems are being investigated as a possible approach to improving the pharmaceutical properties of a candidate drug. By using innovative vectors, such as dendrimers or nanomaterials, it may be possible to improve a drug candidate's bioavailability and reduce its toxic side effects by tailoring the DMPK properties and/or enhancing the uptake and retention of compounds within target organ cells.

Each different delivery vehicle presents the bioanalytical scientist with a unique set of challenges and often there is a requirement not only to understand the drug substance exposure but to also understand the relationship between the drug and its carrier. Indeed, draft regulatory guidance on nanomaterials [23] indicates that exposure data for all relevant entities relating to drug release (e.g., total, free and nanomaterial associated drug) should be measured or indirectly derived. Consensus was reached that the meaning of 'indirectly derived' exposure data, as described in the draft guidance, refers to the measurement of a surrogate for the released drug such as a metabolite. It was also concluded that a mathematical approach for the derivation of drug released from a nanomedicine is likely not feasible due to challenges associated with the relative exposure ranges for each component. The measurement of total drug and encapsulated drug would likely warrant high concentration calibration ranges compared to the assay range required to measure the released concentration which could be within the variability of the total and encapsulated assay ranges.

Nanomedicine formulations are designed to leak or rapidly break down. When the formulation is used to prepare QCs for monitoring of release during sample preparation, there may be differences between documented released amounts of drug in prepared nanomedicines and baseline measurements for QC preparation. There was agreement that stability of the formulation should be taken into consideration when preparing QCs and therefore it is recommended to use the QCs to establish a $\mathrm{T}_{0}$ concentration for the released drug and set the baseline for stability.

To establish the biological fate of the carrier, direct measurement of a single formulation component as a surrogate has been applied in non-clinical study support. One alternative recommended approach could be the use of radiolabelled nanoparticles; some companies using delivery vectors such as lipid nanoparticles monitor both the cationic lipid and the PEG components to characterize their biodistribution.

Nanomaterials interact with multiple plasma proteins resulting in the formation of a protein corona. It was agreed that there has been little experience of the protein corona characterization. However, it was suggested to use proteomic workflows to determine the major binding proteins involved in the formation of the corona.

\section{Innovation in Small Molecules}

Microflow-LCMS Assays

One route to improve the sensitivity of any LCMS platform is to reduce the flow rate through the electrospray ionization source. Compound dependent gains in analyte response have been demonstrated by scaling down column dimensions and reducing the LC flow from $600 \mu \mathrm{l} / \mathrm{min}$ to sub- $10 \mu \mathrm{l} / \mathrm{min}$. To facilitate routine access to these low flow rates, mass spectrometer manufacturers have introduced electrospray ionization sources designed to work at these low flow rates. These sources are designed to easily make zero dead-volume connections to the chromatographic column and to set reproducible alignment of the electrospray emitter with the instrument. With these source designs, sub-10 $\mu \mathrm{l} / \mathrm{min}$ chromatography can be routinely used in a production environment so that $\mathrm{pg} / \mathrm{ml}$ detection limits are routinely obtained and sub-pg/ $\mathrm{ml}$ detections limits are often accessible. This technology is particularly useful for microdosing or low volume studies.

It was agreed that while AMS studies are universally applicable for mass balance studies, the use of microflowLCMS is increasing and due to the potential sensitivity gains should be explored as a replacement to AMS for Phase 0 PK studies, or wherever a highly sensitive assay is needed. While robustness has improved, and at least two commercial options are available, carryover and low throughput remain challenges [25,26]. It was acknowledged that any development that improves the sensitivity of triple-quadrupole mass spectrometry broadens the application of the platform including analysis of exploratory microdosing studies that were once exclusively analyzed using AMS [25,27].

It was agreed that the impact of system dead volume increases significantly at sub- $10 \mu \mathrm{l} / \mathrm{min}$ flow rates resulting in longer assay duty cycles. Furthermore, system dead volume combined with low flow rates through the injection 
valve and system will likely challenge the ability to meet regulatory requirements for system carryover without lengthening the duty cycle further or adding complexity with valving or flushing schemes. Routine use of these systems may be more amenable to the support of non-regulated studies where 'fit-for-purpose' assay requirements for carryover can be more easily met.

\section{Acoustic Dispensing-Mass Spectrometry (AD-MS)}

Significant improvements on both the LC separation and MS instrumentation in the past decade have enabled sub-minute analysis speeds of biological samples. However, it is still one order of magnitude slower than the colorimetry based plate-reader detection that is widely used for high throughput screening (HTS).

Toward the goal of innovation to increase throughput using MS, case studies were presented where the ADE transducer and the standard ESI ion source of a mass spectrometer were coupled through an OPP sampling interface [28]. ADE is a well-established droplet transfer technology that delivers nanoliter droplets with high reproducibility and precision. Screening speeds as fast as 0.4 seconds-per-sample were demonstrated with high sensitivity, high reproducibility, wide linear dynamic range, and broad compound coverage. The continuous-flow of carrier solvent for the OPP maintained ionization stability and actively cleaned the entire flow system resulting in no observed carry-over. More importantly, the data discussed suggest that by limiting the volume of samples to be introduced into the MS (nanoliters), LC separation becomes unnecessary not only for relatively clean matrices (e.g., in vitro enzymatic/cellular systems), but also 'dirty' matrices such as plasma, with minimal sample preparation.

It is noticed that sample dilution seems to be an effective way to mitigate matrix interference, as demonstrated with the plasma/urine sample analysis results. In addition, ion mobility can be leveraged to separate some metabolites (not in all cases, for example, glucuronides). It was agreed that two key challenges of this platform are limited detection sensitivity and lack of selectivity. Sensitivity limitation is due to intrinsically low sample injection volume, nanoliters compared with microliters for conventional LCMS and will be addressed via advancement of hardware (need more sensitive mass spectrometers). The selectivity concern could be troublesome especially for scenarios when metabolites are converted back to parent during ionization (in-source decay) and cannot be resolved by the mass analyzer. However when detection sensitivity is not a limitation and samples are free of tricky metabolism situations, ADE-OPP-MS could be a viable and attractive option. In addition, it was noted that since each well can be analyzed in a fraction of a second it is possible to apply the technology in the discovery setting for high throughput screening up to 100,000 samples per day.

\section{Patient Centric Microsampling}

Patient centric microsampling offers opportunities to minimize sample volume, collect specimens without venipuncture (which may increase patient enrollment opportunities in remote or less developed regions or with episodic or pediatric diseases, provide enhanced data in clinical trials through outpatient sampling, or enable novel data generation).

These new sampling techniques show promise in quantitative PK analysis applications. Success in this space depends on appropriate bioanalytical method development and logistical execution in the clinical trial. Method development may include: 1) obtaining adequate sensitivity for the small sample volume; 2) understanding analyte stability in the dried state accounting for potential extreme situations during sample collection and shipping; 3) assessing the impact of the device on the analytical method; and 4) conducting appropriate validation work. Logistical execution comprises: 1) training the clinical site or patient to ensure sample collection quality and to maintain sample integrity (DNA profiling is one potential means to ensure sample integrity in the at-home setting and data loggers can be used to understand the environmental conditions a sample has been exposed to in transit);2) alleviating patient burden, especially for cases where presenting at a clinical facility can be challenging for the patient (late stage, pediatric patients, and certain diseases); and 3) establishing adjusted workflows for sample handling (e.g., language translation of material, supplies, shipping, data management, etc.). To ensure sample quality in patient driven healthcare, it is imperative that sample collection is simple and straightforward. In addition, if collection occurs outside the clinic, time stamp technology will need to be incorporated. Finally, a development strategy is important for success. Appropriate bridging work must be conducted to understand the PK correlation between wet plasma and dried blood matrices. Sponsors should engage regulators early in development, such as at IND-submission, to solicit feedback on the implementation strategy for novel microsampling approaches. 
Small Molecule Biomarkers by LCMS HRMS for Small Molecule Biomarkers

As discussed in the 2018 White Paper in Bioanalysis [21], in vitro-based CYP and transporter DDI evaluations result in a high number of false positive rates $(\sim 30 \%)$ and categorize new drugs as potential candidates for dedicated clinical DDI evaluations [29]. Rapidly advancing drug transporter [30] and CYP [31] biomarker sciences suggest that selected endogenous compounds, detectable in human plasma and urine, may serve as biomarkers for specific transporters and CYPs. Among them, coproporphyrin isomers [32,33], coproporphyrin-I (CP-I) and coproporphyrinIII (CP-III), bile acids [34], and N1-methylnicotinamide (NMN) [35] have been evaluated as possible candidate biomarkers for organic anion-transporting polypeptides (OATP1B1 and OATP1B3), organic cation transporters (OCT1 and OCT2), and MATEs, respectively. In a similar fashion, 4-beta-hydroxycholesterol (4beta-HC) and 25hydroxycholesterol (25-HC) have been evaluated as possible endogenous biomarkers for CYP3A4 [31]. Application of integrated quantitative/qualitative UHPLC-HRMS workflows for quantitative purposes, especially for endogenous clinical biomarkers, was discussed. Clinical study results to confirm suitability of novel transporter and CYP small molecule biomarkers as a potential substitute for dedicated probe drug DDI studies demonstrate that endogenous biomarkers give the same DDI risk projections as dosing probe drugs. However, this is still an evolving approach and more examples are needed to provide experience to industry and regulators and alleviate any concerns. Many questions still exist around the selectivity for each enzyme, biomarker or specific probe; the dynamic range also remains challenging.

It was agreed that parallelism tests using authentic versus surrogate matrix should be part of the endogenous biomarker qualification/validation plans in order to characterize the matrix with the goal of better understanding the assays. This test for the surrogate analyte may also be important but is not required.

With appropriate language/permissions in the informed consent documents and/or clinical protocols, left over PK samples (e.g., with appropriate storage) can be used for endogenous biomarker profiling or DDI projections. It was recommended that bioanalytical scientists should be involved in writing and interpreting informed consent as much as possible.

\section{Oligonucleotides}

\section{Unique Strategies in Oligonucleotides}

In general, oligonucleotides are classed based on their structure and mechanism of action in miRNA, ASO, siRNA, aptamers and gapmers. For both ASOs and siRNAs, extraction from plasma, urine, CSF and tissue homogenates has focused on AEX-SPE as a generic approach. Hybridization extraction has also been used but since the selection and synthesis of labeled complementary probes is time consuming and expensive, it is not the preferred strategy in the early stages of drug discovery. If a probe is used, the rationale for the design approach should be available.

When using chromatographic-based assays (AEX-HPLC, RP-IP-LCMS), controlling the co-elution of the diastereoisomers while separating the truncated metabolites can potentially be a challenge and should be approached on a case by case basis. As the truncated forms may not be available, it is key to critically evaluate incurred samples with respect to peak width to mitigate the risk of overestimation of full-length levels due to interference of co-eluting truncated metabolites.

It was confirmed that currently when investigating oligonucleotide biodistribution, methodologies that include extraction can also be challenging. Adequate recovery remains a complication; low recovery is acceptable as long as acceptable precision and accuracy is demonstrated.

The discussion regarding metabolites was continued following the recommendations from the 2018 WRIB [21] to question if plasma metabolites were relevant in view of the intracellular action of oligonucleotides. It was recommended to follow the MIST guidance [36] and industry experience [37]. One remaining challenge is that metabolites often distribute to the tissues and there was experience of conducting metabolite measurements in pre-clinical studies, typically in liver, spleen and kidney tissue.

Oligonucleotide shortmers are often only formed in trace amounts. It was recommended to treat shortmers like metabolites; occasionally synthesize where appropriate. 


\section{Recommendations}

Below is a summary of the recommendations made during the $13^{\text {th }}$ WRIB:

1. Toxicology studies with targeted protein degradation can be conducted in a similar manner to small molecules by measuring TK and typical toxicology readouts (e.g., histopathology). If there are findings, mechanistic studies such as off-target activities can be explored on a case by case basis.

2. When measuring a novel therapeutic, mechanistically free, total, and tertiary complexes can be interesting and may be measured in discovery. When performing regulated bioanalysis, studies are mainly conducted in plasma or accessible tissues (e.g., tumor biopsies).

3. The key to RNA-based target therapeutics is the binding selectivity.

4. The meaning of 'indirectly derived' exposure data refers to using metabolites as surrogate for drugs released from nanomaterial formulations. It was also concluded that mathematical approaches to derive the released drug concentration are likely not feasible due to challenges associated with the large differences in method dynamic ranges for total, encapsulated and the released drug whereby the released drug is expected to be within the variability of the total and encapsulated ranges.

5. Since nanomedicines are designed to leak, stability of the nanomedicine formulation should be taken into consideration when preparing QCs to monitor the control of release and therefore it is recommended to use the QCs to establish a $T_{0}$ concentration for the released drug and set the baseline for stability.

6. The use of microflow-LCMS should be explored whenever a highly sensitive method is required, although carryover and low throughput remain challenging. If this technology is to be used in a regulated study, it is important to understand the role of dead volume in order to ensure a robustly developed method.

7. ADE-OPP-MS may be a viable option if the method has the sensitivity and selectivity required for the application. To overcome these challenges, samples can be diluted if matrix interference is an issue and ion mobility can be leveraged to separate some metabolites.

8. Method development of microsampling techniques includes: 1) obtaining adequate sensitivity for the small sample volume; 2) understanding analyte stability in the dried state; 3) assessing the impact of the device on the analytical method; and 4) conducting appropriate validation work.

9. Logistical execution of microsampling comprises: 1) training the clinical site or patient to maintain sample integrity (DNA profiling is one potential means to ensure sample integrity, design of experiments to establish stability in extreme situations); 2) ensuring patient compliance, especially for cases where presenting at a clinical facility can be challenging for the patient (late stage, pediatric patients, and certain diseases); and 3) establishing adjusted workflows for sample handling (e.g., language translation of material, supplies, shipping, data management, etc.).

10. Appropriate bridging work must be conducted to understand the relation of PK in wet plasma to PK in dried blood when microsampling techniques are used. Sponsors should engage regulators early in the development, such as at IND-submission, in order to confirm the work required.

11. Parallelism tests using authentic versus surrogate matrix should be part of the endogenous biomarker qualification/validation plans in order to characterize the matrix with the goal of better understanding the assays. This test for the surrogate analyte may also be important but is not required.

12. Left over PK samples (e.g., with appropriate storage) can be used for endogenous biomarker profiling or DDI projections as long as allowed by the informed consent documents and/or clinical protocols. It was recommended that bioanalytical scientists should be involved in writing and interpreting informed consent as much as possible.

13. If a probe is used for oligonucleotide bioanalysis, the rationale for the design approach should be available. 


\section{SECTION 2 - Mass Spectrometric Method Development Strategies for Large Molecule} Bioanalysis

Surinder Kaur ${ }^{4}$, Renuka Pillutla ${ }^{11}$, Hongbin $\mathrm{Yu}^{12}$, Kevin Bateman ${ }^{1}$, Lorella Di Donato ${ }^{13}$, Shawna Hengel ${ }^{14}$ Wenying Jian ${ }^{15}$, Barry Jones ${ }^{16}$, John Kellie ${ }^{2}$, Anita Lee ${ }^{17}$, Joe Palandra ${ }^{18}$, Natasha Savoie ${ }^{19}$, Petia Shipkova ${ }^{11}$, Susan Spitz ${ }^{20}$, Dian Su${ }^{4}$, Matthew Szapacs ${ }^{2}$, Jian Wang ${ }^{11}$, Katherine Wright ${ }^{18}$, Jianing Zeng ${ }^{11}$

Authors in Section 2 are presented in alphabetical order of their last name, with the exception of the first three authors who were session chairs, working dinner facilitators, major contributors and/or note-takers. Author affiliations can be found at the beginning of the article.

\section{Discussion topics \& consolidated questions collected from the Global Bioanalytical Community}

The following paragraphs report the consolidated questions collected from the Global Bioanalytical Community. Eight discussion topics were extracted from these questions and considered as the most relevant 'hot topics'. They were reviewed by internationally recognized opinion leaders before being submitted for discussion during the $13^{\text {th }}$ WRIB. The background on each issue, discussions, consensus and conclusions are in the next section and a summary of the key recommendations is provided in the final section of this manuscript.

Innovation in Hybrid LBA/LCMS Assays

Orthogonal LBA \& LCMS

Are integrated LBA, protein chemistry and LCMS expertise essential for hybrid LBA/LCMS method development and for combining and interpreting results across multiple LBA \& LCMS platforms? Biotransformations such as oxidation, deamidation and amino acid/subunits clipping can impact safety and efficacy and may or may not be detected in traditional PK assays. Therefore, what additional strategies are required to characterize biotransformation directly? Top down and bottom up? What are the approaches for measuring free, bound, total biotherapeutics? How do you know if biotransformations impact bioanalytical performance? How do you decide which biotransformations should be included as the analytes in bioanalysis? What approaches are used for measuring biotherapeutic, target and biotherapeutic:target complexes? Why is appropriate protein sample handling critical for hybrid LBA/LCMS assays?

\section{Digestion in Regulated Bioanalysis}

Regulatory agencies have raised concerns on the reproducibility of protein digestion for hybrid LBA/LCMS used for regulated studies. What experiments should be performed to ensure digestion is reproducible and meets regulatory requirements? What experiments should be performed to ensure the reference material and the endogenous protein of interest, and target of quantification, digest equivalently in biomarker assays? Are you using an IS to normalize differences in digestion between tubes? If so, how do you choose your IS? What should be included in ICH M10 BMV to ensure there are no issues with the digestion in hybrid LBA/LCMS? What's new from what was discussed in previous WRIB White Papers regarding experience in digestion optimization including enzyme selection and digestion optimization?

Intact Protein HRMS vs Signature Peptide

Is one peptide/one transition suitable for protein quantification? Why or why not? How are data from a confirmatory peptide used? Does this need to be predefined before sample analysis? Should other separation technologies be considered, such as capillary electrophoresis? Are gas phase separations (ion mobility) useful for protein quantification? What are the main barriers to adopting HRMS-based intact protein quantification? What's new from what was discussed in previous WRIB White Papers? Are there examples of where full scan HRMS had a clear advantage over TQMS (i.e., in multiplexing capability, sensitivity, development time) for quantification of known analytes? What is the consensus on advantages of HRMS vs TQMS assay method development time? Is there still agreement upon what was previously discussed in the WRIB White Papers on HRMS [38]? What is the right balance between optimization of acquisition parameters (which might take longer on TQMS) vs optimization of processing parameters (which might take longer on HRMS)? Do groups utilize HRMS and TQMS in parallel for method development and then choose one over the other based on empirical data or is the decision based on other factors? Can and/or should intact mass LCMS quantification replace surrogate peptide LCMS quantification in pre-clinical studies? 


\section{Immunoaffinity \& Sample Preparation}

How much characterization of capture reagents is necessary especially when limited amount of reagents are available? Is there an update on IA experience including hardware and reagent selection and strategy to reduce drug interference in both free and total ligand assays from what was discussed in previous WRIB White Papers [38]? How can you avoid bias in bioanalytical assays: thorough characterization of assay reagents; evaluation of assays and reagents in the context of biotransformation; and definition of relevant bioanalytical endpoints? Capture steps are critical for bioanalytical assays, and must be fully characterized for bias. How do issues occurring in sample preparation, independent of detection methods (i.e., at the capture step) impact the overall assay performance?

\section{Differences between ADC \& PDC}

What are the bioanalytical similarities and differences between ADC and PDC? Is it possible to propose a consolidated bioanalytical workflow for ADC and PDC? What are the challenges of each bioanalytical approach? Are there any other approaches like hybrid LBA/LCMS for total antibody, DAR based quantification or intact LCMS quantification assay for conjugate? For modifications such as linker succinimide ring hydrolysis or payload biotransformation which may or may not affect potency, is it needed to quantify the concentrations? What are the challenges? For highly engineered ADCs/PDCs constructed with stable drugs and linkers conjugated to specific sites, the measurement of total antibody/protein and conjugate should give identical results. In this case, will fewer assays be acceptable?

\section{Biomarker Assays}

Absolute Accuracy for Biomarker Assays

Can the AQUA technique be a suitable approach in bioanalysis? Can we establish reliable bioanalytical acceptance criteria for quantitative assays for biomarkers by applications of AQUA in regulated bioanalysis? Is AQUA just for proteomics applications? What are AQUA limitations as a targeted quantitative technique for proteins and their modification states? What criteria can be used in bioanalysis (but not proteomics) for surrogate peptide synthesis with incorporated stable isotopes as internal standards to mimic native peptides formed by digestion? How can the industry work together to overcome the lack of absolute accuracy for biomarker LCMS and hybrid LBA/LCMS assays not having an appropriate or exact reference standard? Can the use of the AQUA technique help to reach absolute accuracy? Can the AQUA technique ever be accepted by regulators for biomarkers endpoints in clinical trials?

\section{Tissue Protein Biomarkers}

How can the challenges in quantitative analyses of tissue-bound proteins be overcome when the target is not soluble or readily shed? What are the issues in the correlation of the target level measured by LBA \& LCMS orthogonal approaches? How can the post dosing information of target modification from LBA \& LCMS be combined? What are the standard practices in high sensitivity-based measurements? What, if any, are the current limitations preventing greater usage of nanoflow LC or peptide IA? What are the current standard approaches for normalizing tissue-based measurements? Should we choose one over the other? How can the sensitivity challenge be overcome with the measurement of low concentration analytes in tumor? What is the best positive control for validation? What are the strategies for getting good quality tumor for assessments when quantity (core biopsies) can be limited? Is tumor/tissue concentration data expected for regulatory submission? Are all the analytes required? Are additional analytes such as active catabolites required?

\section{Free Biomarker Assays}

What constitutes a good QC sample for free assay qualification: exogenous binding of drug and recombinantprotein standard; exogenous binding of drug and endogenous target; measuring an expected $\mathrm{EC}_{50}$ from frozen $\mathrm{QC}$ samples? How much variability can be tolerated for compliance? Is it possible to truly measure free target? What is the context around the measurement and the transparent limitations of our bioanalysis methods? How can the expectation that this can be an absolute measurement be managed? 
Discussions, consensus \& conclusions

Innovation in Hybrid LBA/LCMS Assays

Orthogonal LBA \& LCMS

Over the past several years there has been a gradual merging of two previously distinct and separate technological areas in bioanalysis - LBA and LCMS. This mirrors the evolution of complex therapeutic modalities and increasingly complicated disease areas being tackled. There is increased recognition that these two technological platforms are in many ways complementary and can help answer challenging questions in drug discovery and development. For example, correlating data obtained from LBA and LCMS can help understand dose-dependent drug exposure, interferences from $\mathrm{ADA}$ and/or target and correlation to efficacy and safety.

Integrated LBA, protein chemistry, and LCMS expertise are essential for hybrid LBA/LCMS method development and for combining and interpreting results across multiple LBA and LCMS platforms. It was recommended that in order to build expertise in the combined platforms, proficiency in each individual platform is needed.

If a given biotransformation has the potential to affect the structure, function, safety, efficacy (e.g., ADCs) or the performance of the bioanalytical assay, then the assessment is relevant. Application of the appropriate methodologies can help characterize biotransformation such as in vivo oxidation, isomerization and proteolytic degradation. Oxidation, deamidation and amino acid/subunits clipping can impact safety and efficacy and may or may not be detected in traditional PK assays depending on the capture and/or detection approach used. Therefore, additional strategies may be required to characterize relevant biotransformation directly (i.e., top down and bottom up). It was recommended that any assessments be driven by the scientific questions that need to be addressed. Assays and data should not be generated just because it is technically feasible. The biochemistry and function of the molecule should drive the need. The scientific need for the assay should be carefully considered. Having a clear scientific rationale is important prior to designing and executing complex biotransformation characterization analyses.

For measurements of circulating biomarkers, LBA and LCMS can be applied to distinguish between free, complexed and total target. It was recommended to define and understand what the assay is measuring to provide support for the claim and to obtain meaningful and interpretable data.

Appropriate protein sample handling techniques are critical for hybrid LBA/LCMS assays. Any improperly stored and handled samples and reagents, can cause an assay to under-perform, fail or provide incorrect results.

\section{Digestion in Regulated Bioanalysis}

Quantification of protein biomarkers is essential to provide an understanding of PD parameters for a given therapeutic and is critical for safety and efficacy of drug in development. LBAs have traditionally been the primary means for quantification of protein biomarkers but are dependent on high quality capture and detection antibodies to develop an assay with the required sensitivity and specificity. In recent years, analytical methods utilizing LCMS have been developed and validated as alternatives to LBAs; LCMS is less dependent on capture reagent quality, provides a high degree of specificity, can distinguish multiple protein isoforms, and is often easier to multiplex compared to LBAs. LCMS methods for the quantification of protein biomarkers typically utilize an enzymatic digestion step that cleaves proteins into peptides that are amenable to LCMS workflows and generally allow for more sensitive assays than intact protein analysis. However, the use of a digestion step in protein biomarker assays can be complicated compared to PK assays, given that the protein standard is typically recombinant (reference material) for the preparation of calibration standards and measured in a surrogate matrix. It is possible that the protein test standard and endogenous protein may exhibit different digestion kinetics if the molecular structures are sufficiently different (e.g., different levels of glycosylation). As the digestion step is critical to providing a robust and reproducible assay, an attempt should be made to have a thorough understanding of this step for both the reference material and endogenous biomarker protein. However, as there are several conditions that can be optimized prior to or during the digestion step (including temperature, reduction or alkylation steps, denaturants, enzyme choice, protein to enzyme ratio and microwave or pressure assisted digestion), a systematic approach to assessing and optimizing digestion efficiency is required for rapid assay development and subsequent validation.

Regulators have raised concerns on the reproducibility of protein digestion for hybrid LBA/LCMS used for regulated PK studies [39]. To ensure that the digestion step is reproducible and meets regulatory requirements, it was recommended to demonstrate adequate precision of samples representative of the study population. Complete digestion is not required, but it must be reproducible and not concentration dependent. 
The use of an IS to normalize differences in digestion between intra- and inter-day replicates was discussed. To perform such normalization, the IS must be at the protein level that is either a heavy-labeled protein or analogue protein. Attendees stated that they typically did not use this approach, but when an IS was used, it was rare that differences were detected. Labeled protein IS is not always available though stable isotope-labeled universal $\lg G$ monoclonal antibody SILu ${ }^{T M} \mathrm{mAb}$ can be used for PK study analysis. Taking the approach of optimizing assay conditions made a bigger difference than relying on the presence of an IS, especially when the IS used is at the peptide level. However, if a new enzyme is used for digestion or if there are concerns with differences in digestion efficiency from sample to sample (i.e., hemolyzed or lipaemic) then the use of an IS at the protein level has proven to be useful.

Experience in enzyme selection has increased from what was discussed in previous WRIB White Papers [38]. Current recommendations include the use of a trypsin that provides reproducible results across lots. Newer forms of trypsin, such as high temperature trypsins and rapid trypsins are becoming more popular to reduce the timing of the digestion step.

\section{Intact Protein HRMS vs Signature Peptide}

Increased complexity of protein biotherapeutics has driven improvements in bioanalytical techniques to support this sector of drug development. The most prevalent LCMS approach for biotherapeutic quantification is built on protein digestion followed by SRM-based quantitative analysis. Digestion provides unique signature analytes for different parts of the larger intact version which allows for more standardized LC and MS methodology to be used. This approach has proven effective but can theoretically raise questions about what is actually being measured, as the digestion-based surrogate peptide approach covers a low percentage of the total amino acid sequence. It was discussed whether intact bioanalysis using HRMS has an advantage over traditional bottom-up approaches, but it was concluded that the advantages of intact analysis is currently limited by the complex data processing needed and the lack of sensitivity. Intact analysis may be used as a complementary technique dependent on the stage of development, the question being asked and the analyte - for example, to confirm the structural integrity of large molecules quantified using enzymatic digestion approaches.

The 2018 White Paper in Bioanalysis [20] highlighted some of these challenges and recommended an intact mass based approach for protein quantification. For instance, it was confirmed that intact mass LCMS quantification could replace surrogate peptide LCMS quantification in pre-clinical studies for a regular $\mathrm{mAb}$, where high sensitivity is not typically required. However, based on 2019 discussions, there remain challenges with the intact mass approach, such as sensitivity, chromatography, throughput, and the need for more robust data processing software. Future developments in chromatography and software may make intact MS for quantitation more readily applicable.

If the signature peptide approach is used for protein quantification it is important to carefully select and predefine the peptide or peptides to be used. For PK assays, at the assay development stage it is recommended to monitor several peptides and several transitions per peptide. At the end of development prior to assay validation, it is recommended to identify the best peptide or peptides and best transitions for the peptide. For protein biomarkers, assay development is also performed by monitoring several peptides and several transitions per peptide. Prior to assay validation, the two best peptides are selected and two transitions per peptide are monitored. For the two peptides it is recommended to identify the peptide that will be used to report data and the peptide that will be used for monitoring purposes.

\section{Immunoaffinity \& Sample Preparation}

With the recent advances in IA approaches and techniques, hybrid LBA/LCMS has been extensively used for the measurement of protein therapeutics [40] and protein biomarkers. Combining advantages of both LBA and LCMS technology, hybrid LBA/LCMS approach offers high assay selectivity, robust assay performance, and multiplexing capability. It utilizes non-specific and/or specific capture reagents to isolate and enrich the target analytes from biological matrices, followed by targeted LCMS detection, of either enzymatic surrogate peptide(s) or the intact protein using either triple quadrupole or high-resolution mass spectrometry. Highly selective IA enrichment in a hybrid LBA/LCMS approach greatly removes the sensitivity obstacle for the LCMS based assays and makes the hybrid LBA/LCMS approach an attractive methodology for the quantification of low abundance proteins. This topic was revisited for updated recommendations on previous WRIB White Papers [38]. Consensus was that although there was more rigor occurring with this platform, previous recommendations are still applicable. 
One of the issues facing the use of the IA approach is the amount of characterization needed for the capture reagent, especially when a limited amount of the material is available. The level of characterization is dependent on the type of reagent. It was deemed important to fully characterize the capture reagent for PK assays. However, for biomarker assays with limited reagents, then it was recommended to perform as much characterization as feasible.

Capture steps are critical for all bioanalytical assays and must be fully characterized for bias [41]. It was recommended to investigate the binding capacity and interference of the assay. The use of a full-length labeled protein as an IS would be a benefit when using a capture step. Preclinically, this is facilitated by the availability of commercial generic mAb IS. Furthermore, in the biomarker space, it is important to investigate whether the recombinant protein binds differently than the endogenous target.

\section{Differences between $A D C$ \& $P D C$}

Thorough bioanalytical packages tailored to support ADC programs require extensive development, specific reagent generation, and multi-analyte interference/stability testing across multiple assays. Bioanalytical techniques are often selected in part through sensitivity or selectivity requirements, in addition to linker chemistries, conjugated drug, and disease indication driving the nuances of the assays. While most method development occurs using characterized reference standards, one must anticipate how biotransformation, at the antibody, drug linker, and conjugated drug levels, can impact assay readouts and PK analyses. Thorough investigation of the kinetics of change and definition of which analytes require quantification, or characterization, using bioanalytical assays aid in selecting assay platforms.

Many ADCs currently in the clinic undergo drug loss in circulation -that is, deconjugation of the linker through retro-Michael reactions or release of the conjugated drug, which shifts the drug load distribution in circulation. Characterization of reagents and assays used to quantify ADCs with a distribution of drug loading that is susceptible to change over time provides robust data sets and minimizes assay redevelopment. Specifically, assays which utilize capture steps including ELISA and hybrid LCMS methods can be evaluated for binding or recovery with a range of drug loaded ADCs that are predicted to be in circulation in vivo.

Alternative scaffold proteins have emerged as a novel platform for the development of therapeutic applications and particularly for use in targeted delivery. One such application is in PDCs, which are analogous to the ADC platform. To fully describe the PK and disposition of a PDC in vivo, concentrations of total protein, conjugate, and free drug in plasma and tissue samples all need to be determined. It was agreed that the PDC assay workflow could be simpler than for ADC because the smaller size and simpler structures of typical PDCs. It is possible to apply hybrid LCMS to measure total protein and conjugate in a multiplexed assay, and intact LCMS analysis is also more amenable for PDCs.

For highly engineered ADCs/PDCs constructed with stable drugs and linkers conjugated to specific sites, the measurement of total antibody/protein and conjugate should give identical results. Consensus was that fewer assays may ultimately be needed for a given program if this assay equivalence is demonstrated early on.

\section{Biomarker Assays \\ Absolute Accuracy for Biomarker Assays}

The biomarker quantification approach known as AQUA makes use of synthetic peptides that contain SIL amino acids $(13 \mathrm{C}, 15 \mathrm{~N})$ for which their synthesis is based on the sequence of the peptides of interest [42]. Although it is named the absolute quantification approach, it is truly measuring relative accuracy. It is called 'absolute' because the SIL-peptide is well characterized. However, it remains relative because the SIL-peptide does not undergo the enzymatic digestion as the endogenous protein and a calibration standard curve approach is not used. These peptides are spiked into the sample (most often at the post-digestion step) at a fixed concentration to measure the endogenous levels of the peptides as surrogates to the respective endogenous proteins. The endogenous levels of the peptide of interest are obtained by measuring the ratio of the peak area of the endogenous (native) to SIL-peptide. It is by using the ratio and the concentration of the SIL-peptide added to the sample that the endogenous levels are determined. This is most useful in highly multiplexed biomarker assays and in tissue matrices for which spiking recombinant proteins in this type of matrix is a challenge. In contrast, this approach is not appropriate for a PK assay since the drug product administered to the subjects is also the reference material used to determine its respective concentration in the subjects. The measurement of drug product (PK assay) is thereby an absolute quantitation.

The need to provide absolutely accurate data for fit-for-purpose biomarker assays, based on surrogate recombinant calibration standards, was discussed. For example, there are validation experiments such as carryover and specificity that cannot be conducted based on a surrogate recombinant reference standard. It was concluded that absolute 
accuracy may not be necessary if the results provide sufficient information to answer the scientific question posed. If the biomarker is selected as a PD marker later in development, assay robustness should be adequately assessed.

\section{Tissue Protein Biomarkers}

Modern drug discovery is quickly adapting to a new paradigm with greater emphasis placed on translational research and specifically target quantification. While measurements of soluble targets are well established and routinely done in drug discovery and development, quantitative analyses of tissue-bound proteins are relatively new and not often performed. Such measurements are particularly imperative in cases where the target is not soluble or readily shed and characterization of target tissue distribution and expression is needed.

In pre-clinical studies, tissue samples are typically obtained by resection and are much larger than average clinical tissue samples, often collected as needle biopsies. The availability of sufficient samples for method development allows assessment and optimization of sample handling, extraction, analyte stability and analysis reproducibility. In an ideal case, prior to finalizing a clinical biomarker plan and clinical sample analyses, relevant pre-clinical experiments are conducted evaluating the tissue target/ligands of interest. This can only be successfully accomplished when there is sufficient understanding of tissue handling under different collection protocols and treatments.

Optimization of tissue extraction, enrichment, digestion, and normalization are all extremely important and necessary factors for quick and successful development of biomarker LCMS assays. Availability of orthogonal measurements such as a LBA, flow cytometry or immuno-histochemistry staining, can provide an additional level of confirmation and confidence in progressing new targets and drugs for treatment of various diseases. In order to normalize tissue-based measurements, total protein is widely used but comes with challenges.

To overcome the challenges in quantitative analyses of tissue-bound proteins, different combinations of detergents and lysates that do not destroy the protein should be used. Where this is not possible, it was suggested that capturing at the peptide level may be appropriate. The reagent needs are the key to the determination of the ideal platform. The best positive control for validation was recommended to be pooled lysate.

Nanoflow LC or peptide IA may be options for highly sensitive measurements but have several challenges. For instance, the learning curve to using these techniques is steep, the capture antibody for the peptide may not be readily available and sample cleanliness may be a limitation without additional online IA column enrichment. Therefore, for these reasons, nanoflow or peptide IA is primarily used in a discovery based environment, as opposed to a regulated one however this is an evolving area where CROs are developing skills to support these assay types.

\section{Free Biomarker Assays}

Over the last decade, biologics are playing larger roles in pharmaceutical discovery pipelines. With more preclinical support utilizing hybrid LBA/LCMS assays, the natural progression of the technique is extended to include clinical sample analysis for consistency across the discovery to development process. With this extension of support comes the need to develop more complex assays to measure total and free target for dose projection modeling and clinical sample support to ensure target coverage.

It was discussed what makes a good QC sample for free assay qualification. The exogenous binding of drug and recombinant-protein standard or the exogenous binding of drug and endogenous target could be used depending on concentration of the biomarker. Tolerance limits of variability should be fit for purpose. It was clarified that when measuring the free target, the measurement is relative, not absolute.

\section{Recommendations}

Below is a summary of the recommendations made during the $13^{\text {th }}$ WRIB:

1. To build expertise in hybrid LBA/LCMS platforms, proficiency in each individual platform is needed.

2. If a biotransformation affects the structure, function, safety, efficacy (e.g., ADCs), or the performance of the bioanalytical assay, then an assessment is necessary. Assuming biotransformations are relevant, additional strategies may be required to characterize biotransformation directly (i.e., top down and bottom up), although this should not be a routine assessment. Knowledge of protein stability provided by Chemistry, Manufacturing and Controls colleagues is important to determine whether biotransformation investigation is warranted. The need for the assay should be considered. Having a clear scientific rationale is important.

3. Understand if the assay is measuring free, complexed or total target to provide support for the claim and to interpret the data in the context of what is being measured. 
4. Appropriate protein sample and reagent handling is critical for hybrid LBA/LCMS assays.

5. To ensure digestion is reproducible and meets regulatory requirements, it was recommended to demonstrate adequate precision of samples representative of the study population. Complete digestion is not required, but it must be reproducible. Intact labeled IS or extended length SIL peptides can be useful when available.

6. Optimization of hybrid LBA/LCMS assay conditions may have a larger impact than relying on the presence of an IS, especially when the IS used is at the peptide level. However, if a new enzyme is used for digestion, additional characterization of digestion efficiency from sample to sample (i.e., hemolyzed or lipaemic) is warranted. The use of an IS at the protein level has proven to be useful.

7. Current recommendations in digestion optimization include the use of newly developed enzyme products with higher digestion efficiency such as high temperature trypsin and rapid trypsin.

8. Intact protein analysis by HRMS may be used as a complementary technique to traditional bottom-up LCMS based on the stage of development, the question being asked and the analyte.

9. Intact mass LCMS quantification could replace surrogate peptide LCMS quantification in preclinical studies for a regular $\mathrm{mAb}$ where high sensitivity is not required.

10. If the signature peptide approach is used for protein quantification it is important to carefully select and predefine the peptide to be used. For PK assays, at the assay development stage it is recommended to monitor several peptides and several transitions/peptide.

11. Consensus was that although there was more rigor occurring with the hybrid LBA/LCMS platform, previous WRIB White Paper recommendations [20,38] are still applicable.

12. The amount of characterization needed for the capture reagent is dependent on the type of reagent. It was deemed important to fully characterize the capture reagent for PK assays (e.g., binding affinity, monomer content, assay performance, etc.). However, for biomarker assays with limited reagents, it was recommended to perform as much characterization as feasible.

13. It was recommended to investigate the binding capacity and interference of the hybrid LBA/LCMS assay. In the biomarker space, it is important to investigate whether the recombinant protein binds differently than the endogenous target. Use of full-length labeled protein as IS when available is recommended.

14. To fully describe the PK and disposition of a protein-drug conjugate in vivo, concentrations of total protein, conjugate, and free payload in plasma and tissue samples all need to be determined. It was agreed that the PDC assay workflow could be simpler than for ADC.

15. For PDC and ADC, fewer assays would be acceptable later on if it is proven early that the measurement of total antibody/protein and conjugate give identical results for a given species.

16. The AQUA approach is not appropriate for a PK assay, but is most useful in highly multiplexed biomarker assays and also in tissue matrices for which spiking recombinant proteins in this type of matrix is a challenge.

17. Absolute accuracy may not be necessary for biomarker assays if the results provide enough information to answer the question posed. If the biomarker is later selected as a PD marker, assay robustness should be fully assessed.

18. To overcome the challenges in quantitative analyses of tissue-bound proteins, different combinations of detergents and lysates that do not destroy the protein should be used.

19. Nanoflow is typically only used regularly in discovery for low-level circulating and tissue biomarkers, more rarely for regulated bioanalysis.

20. The best positive control for tissue protein biomarker validation was recommended to be a pooled lysate.

21. The exogenous binding of drug and recombinant-protein standard or the exogenous binding of drug and endogenous target could be used as a QC depending on concentration of the biomarker. Tolerance limits of variability should be fit for purpose.

22. The free target measurement is relative, not absolute.

\footnotetext{
Acknowledgements

- The US FDA, Europe EMA, UK MHRA, Brazil ANVISA, Health Canada, Japan MHLW, France ANSM and Norway NoMA for supporting this workshop;

- Booth B (US FDA), Fandozzi C (Merck), Evans C (GlaxoSmthKline), Pillutla R (Bristol-Myers Squibb), Kaur S (Genetech), Yu H (Boeringer Ingelheim), Garofolo F (Angelini Pharma), Schweighardt B (BioMarin), Liang M (AstraZeneca), Stevenson L (Biogen); Buonarati M (Inertek), Vitaliti A (Novartis), Litwin V (Caprion), Mehta D (Biogen), Piccoli S (GlaxoSmithKline),
} 
Gorovitz B (Pfizer), Palmer R (Sanofi), Amavaradi L (Shire/Takeda), Beaver C (Syneos), Richards S (Sanofi), Rajadhyaksha M (Regeneron) for chairing the workshop and/or the White Paper discussions;

- All the workshop attendees and members of the Global Bioanalytical Community who have sent comments and suggestions to the workshop to complete this White Paper;

- Future Science Group as a trusted partner.

\section{Financial \& competing interests disclosure}

The authors have no relevant affiliations or financial involvement with any organization or entity with a financial interest in or financial conflict with the subject matter or materials discussed in the manuscript. This includes employment, consultancies, honoraria, stock ownership or options, expert testimony, grants or patents received or pending, or royalties.

No writing assistance was utilized in the production of this manuscript.

\section{References}

1 Savoie N, Booth BP, Bradley T et al. 2008 White Paper: The 2nd Calibration and Validation Group Workshop on Recent Issues in Good Laboratory Practice Bioanalysis. Bioanalysis 1(1), 19-30 (2009).

2 Savoie N, Garofolo F, van Amsterdam P et al. 2009 White Paper on Recent Issues in Regulated Bioanalysis from the 3rd Calibration and Validation Group Workshop. Bioanalysis 2(1), 53-68 (2010).

3 Savoie N, Garofolo F, van Amsterdam P et al. 2010 White Paper on Recent Issues in Regulated Bioanalysis and Global Harmonization of Bioanalytical Guidance. Bioanalysis 2(12), 1945-1960 (2010).

4 Garofolo F, Rocci M, Dumont I et al. 2011 White Paper on Recent Issues in Bioanalysis and Regulatory Findings from Audits and Inspections. Bioanalysis 3(18), 2081-2096 (2011).

5 DeSilva B, Garofolo F, Rocci M et al. 2012 White Paper on Recent Issues in Bioanalysis and Alignment of Multiple Guidelines. Bioanalysis 4(18), 2213-2226 (2012).

6 Stevenson L, Rocci M, Garofolo F et al. 2013 White Paper on Recent Issues in Bioanalysis: "Hybrid” - the best of LBA \& LC/Ms. Bioanalysis 5(23), 2903-2918 (2013).

7 Fluhler E, Hayes R, Garofolo F et al. 2014 White Paper on Recent Issues in Bioanalysis: a full immersion in bioanalysis (Part 1 - small molecules by LCMS). Bioanalysis 6(22), 3039-3049 (2014).

8 Dufield D, Neubert H, Garofolo F et al. 2014 White Paper on Recent Issues in Bioanalysis: a full immersion in bioanalysis (Part 2 hybrid LBA/LCMS, ELN \& regulatory agencies' input). Bioanalysis 6(23), 3237-3249 (2014).

9 Stevenson L, Amaravadi L, Myler H et al. 2014 White Paper on Recent Issues in Bioanalysis: a full immersion in bioanalysis (Part 3 LBA and immunogenicity). Bioanalysis 6(24), 3355-3368 (2014).

10 Welink J, Fluhler E, Hughes N et al. 2015 White Paper on Recent Issues in Bioanalysis: focus on new technologies and biomarkers (Part 1 - small molecules by LCMS). Bioanalysis 7(22), 2913-2925 (2015).

11 Ackermann B, Neubert H, Hughes N et al. 2015 White Paper on Recent Issues in Bioanalysis: focus on new technologies and biomarkers (Part 2 - hybrid LBA/LCMS and input from regulatory agencies). Bioanalysis 7(23), 3019-3034 (2015).

12 Amaravadi L, Song A, Myler H et al. 2015 White Paper on Recent Issues in Bioanalysis: focus on new technologies and biomarkers (Part 3 - LBA, biomarkers and immunogenicity). Bioanalysis 7(24), 3107-3124 (2015).

13 Yang E, Welink J, Cape S et al. 2016 White Paper on Recent Issues in Bioanalysis: focus on biomarker assay validation (BAV) (Part 1 small molecules, peptides and small molecule biomarkers by LCMS). Bioanalysis 8(22), 2363-2378 (2016).

14 Song A, Lee A, Garofolo F et al. 2016 White Paper on Recent Issues in Bioanalysis: focus on biomarker assay validation (BAV): (Part 2 Hybrid LBA/LCMS and input from regulatory agencies). Bioanalysis 8(23), 2457-2474 (2016).

15 Richards S, Amaravadi L, Pillutla R et al. 2016 White Paper on Recent Issues in Bioanalysis: focus on biomarker assay validation (BAV): (Part 3 - LBA, biomarkers and immunogenicity). Bioanalysis 8(23), 2475-2496 (2016).

16 Welink J, Yang E, Hughes N et al. 2017 White Paper on Recent Issues in Bioanalysis: aren't BMV guidance/guidelines 'scientific'? (Part 1 - LCMS: small molecules, peptides and small molecule biomarkers). Bioanalysis 9(22), 1807-1825 (2017).

17 Neubert H, Song A, Lee A et al. 2017 White Paper on Recent Issues in Bioanalysis: rise of hybrid LBA/LCMS immunogenicity assays (Part 2: hybrid LBA/LCMS biotherapeutics, biomarkers \& immunogenicity assays and regulatory agencies' inputs). Bioanalysis 9 (23), 1895-1912 (2017).

18 Gupta S, Richards S, Amaravadi L et al. 2017 White Paper on Recent Issues in Bioanalysis: a global perspective on immunogenicity guidelines \& biomarker assay performance (Part 3 - LBA: immunogenicity, biomarkers and PK assays). Bioanalysis 9 (24), 1967-1996 (2017).

19 Welink J, Xu Y, Yang E et al. 2018 White Paper on Recent Issues in Bioanalysis: 'a Global Bioanalytical Community perspective on last decade of incurred samples reanalysis (ISR)' (Part 1 - small molecule regulated bioanalysis, small molecule biomarkers, peptides $\&$ oligonucleotide bioanalysis). Bioanalysis 10(22), 1781-1801 (2018). 
20 Neubert H, Olah T, Lee A et al. 2018 White Paper on Recent Issues in Bioanalysis: focus on immunogenicity assays by hybrid LBA/LCMS and regulatory feedback (Part $2-$ PK, PD \& ADA assays by hybrid LBA/LCMS \& regulatory agencies' inputs on bioanalysis, biomarkers and immunogenicity). Bioanalysis 10(23), 1897-1917 (2018).

21 Stevenson L, Richards S, Pillutla R et al. 2018 White Paper on Recent Issues in Bioanalysis: focus on flow cytometry, gene therapy, cut points and key clarifications on BAV (Part 3 - LBA/cell-based assays: immunogenicity, biomarkers and PK assays). Bioanalysis 10(24), 1973-2001 (2018).

22 ICH M10, Draft Bioanalytical Method Validation (2019). www.ich.org/fileadmin/Public_Web_Site/ICH_Products/Guidelines/Multidisciplinary/M10/M10EWG_Step2_DraftGuideline_2019_0226.pdf

23 US FDA, Draft Guidance for Industry. Drug Products, Including Biological Products that Contain Nanomaterials (2017). www.fda.gov/media/109910/download

24 Howe JA, Wang H, Fischmann TO et al. Selective small-molecule inhibition of an RNA structural element. Nature. 29, 526(7575), 672-677 (2015).

25 Burt T, John CS, Ruckle JL, Vuong LT. Phase-0/microdosing studies using PET, AMS, and LC-MS/MS: a range of study methodologies and conduct considerations. Accelerating development of novel pharmaceuticals through safe testing in humans - a practical guide. Expert Opinion on Drug Delivery 14(5), 657-672 (2017).

26 Yuan L, Huang C, Liu-Kreyche P et al. A convenient strategy to overcome interference in LC-MS/MS analysis: application in a microdose absolute bioavailability study. J. Pharm. Biomed. Anal. 165, 198-206 (2019).

27 Jiang H, Zeng J, Li W et al. Practical and efficient strategy for evaluating oral absolute bioavailability with an intravenous microdose of a stable isotopically-labeled drug using a selected reaction monitoring mass spectrometry assay. Anal. Chem. 84, 10031-10037 (2012).

28 Zhang H. Acoustic dispensing-mass spectrometry: the next high throughput bioanalytical platform for early drug discovery. Bioanalysis 9(21), 1619-1621 (2017).

29 Vaidyanathan J, Yoshida K, Arya V, Zhang L. Comparing various in vitro prediction criteria to assess the potential of a new molecular entity to inhibit organic anion transporting polypeptide 1B1. J. Clin. Pharmacol. 56(Suppl. 7), S59-S72 (2016).

30 Rodrigues AD, Taskar KS, Kusuhara H, Sugiyama Y. Endogenous probes for drug transporters: balancing vision with reality. Clin. Pharmacol. Ther. 103(3), 434-448 (2018).

31 Nitta SI, Hashimoto M, Kazuki Y et al. Evaluation of 4beta-hydroxycholesterol and 25-hydroxycholesterol as endogenous biomarkers of CYP3A4: study with CYP3A-humanized mice. AAPS J. 20(3), 61 (2018).

32 King-Ahmad A, Clemens S, Ramanathan R et al. A fully automated and validated human plasma LC-MS/MS assay for endogenous OATP biomarkers coproporphyrin-I and coproporphyrin-III. Bioanalysis 10(9), 691-701 (2018).

33 Njumbe Ediage E, Dillen L, Vroman A et al. Development of an LC-MS method to quantify coproporphyrin I and III as endogenous biomarkers for drug transporter-mediated drug-drug interactions. J. Chromatogr. B Analyt. Technol. Biomed. Life Sci. 1073, 80-89 (2018).

34 Rago B, Tierney B, Rodrigues AD, C LH, Ramanathan R. A multiplex HRMS assay for quantifying selected human plasma bile acids as candidate OATP biomarkers. Bioanalysis 10(9), 645-657 (2018).

35 Luo L, Kay J, Zhang J et al. LC-MS/MS assay for N(1)-methylnicotinamide in humans, an endogenous probe for renal transporters. Bioanalysis 10(9), 673-689 (2018).

36 US FDA. Guidance for Industry: Safety Testing of Drug Metabolites (2008). www.fda.gov/media/72279/download

37 Schadt S, Bister B, Chowdhury SK et al. A Decade in the MIST: learnings from investigations of drug metabolites in drug development under the "Metabolites in Safety Testing" regulatory guidance. Drug Metab Dispos 46(6), 865-878 (2018).

38 Garofolo W, Savoie N. The Decennial Index of the White Papers in Bioanalysis: 'A Decade of Recommendations (2007-2016)'. Bioanalysis 9(21), 1681-1704 (2017).

39 Booth B, Furmanski B. Hybrid assays: the next big thing? Bioanalysis 10(13), 975-977 (2018).

40 Kaur S, Liu L, Cortes DF, Shao J, Jenkins R, Mylott WR Jr, Xu K. Validation of a biotherapeutic immunoaffinity-LC-MS/MS assay in monkey serum: 'plug-and-play' across seven molecules. Bioanalysis 8(15), 1565-1577 (2016).

41 Liu L, Xu K, Li J et al. Optimizing hybrid LC-MS/MS binding conditions is critical: impact of biotransformation on quantification of trastuzumab. Bioanalysis 10(22), 1819-1831 (2018).

42 Ye X et al. Targeted proteomics for validation of biomarkers in clinical samples, Brief Funct. Genomic Proteomic. 8(2), 126-135 (2009). 